\title{
Assisted Channel Estimation with Dynamic Allocation Pilot Subcarriers for OFDM Communications
}

\author{
Boudali Ouarzazi $^{1}$, Atika Menhaj-Rivenq ${ }^{1}$, Iyad Dayoub ${ }^{1}$, Marion Berbineau ${ }^{2}$ \\ ${ }^{1}$ Department OAE, Institut d'Electronique de Microelectronique et de Nanotechnologie, Université de Valenciennes et du Hainaut- \\ Cambrésis, Valenciennes, France; ${ }^{2}$ Université Lille Nord de France, Villeneuve d'Ascq, France. \\ Email: name.familyname@univ-valenciennes.fr
}

Received June $10^{\text {th }}, 2013$; revised July $19^{\text {th }}, 2013$; accepted August $2^{\text {nd }}, 2013$

Copyright (C) 2013 Boudali Ouarzazi et al. This is an open access article distributed under the Creative Commons Attribution License, which permits unrestricted use, distribution, and reproduction in any medium, provided the original work is properly cited.

\begin{abstract}
In this paper, a transmission system based on OFDM (Orthogonal Frequency Division Multiplexing) technique and channel estimation is proposed. The idea is to enhance the assisted channel estimation without data rate loss. A solution based on frequency diversity is proposed. With the same number of pilots, the global system performances are better for higher mobility speed. The main results will be presented in the case of high mobility context.
\end{abstract}

Keywords: Doppler Effect; Channel Estimation; OFDM; Rayleigh Channel; BEM; Mobility

\section{Introduction}

The channel estimation is a fundamental step in a transmission system whose performances are directly related to the severity of this step in the reception process. The estimation quality is highly dependent on variations of the channel propagation. In the literature, one can find three main types of channel estimation techniques: supervised or assisted techniques, blind techniques and semiblind techniques with decision feedback [1]. The blind estimation technique does not require insertion of pilot subcarriers in the frame. It uses some statistical properties of the received signal (i.e. redundancy due to the insertion of cyclic prefix, higher order statistics) and directly addresses the problem of loss of spectral efficiency [2-5]. However, this technique often requires significant computation time and isn't appropriate for this case of high mobility. The estimation technique with a semiblind decision feedback using both known data (i.e. pilot subcarriers) and estimated data sent with a forward link. This estimation is very sensitive to the detection of errors on the estimated symbols $[1,4]$, and in particular in the context of the high mobility with rapid channel variations. The assisted channel estimation is based on inserting, in the OFDM frame, symbols which are known by the receiver and called pilot symbols. The associated subcarriers are called the subcarrier pilots. Training sequence corresponds to the set of pilot subcarriers dedicated to the channel estimation. It consists in a pattern robust to the frequency selectivity of the channel. It is suitable for quasi-stationary channels for which the channel coefficients vary little from one OFDM symbol to another. The insertion of pilot "comb type" consists in reserving some pilot subcarriers only to channel estimation for all transmission duration. This pattern is robust to the channel temporal variations from an OFDM symbol to another [6]. The main disadvantage of the channel assisted estimating technique is the spectral efficiency loss due to the insertion of pilots between the transmitted data. However, we chose this technique despite the loss of spectral efficiency. In the broadband challenge for high mobility, the hostile characteristics nature of mobile propagation channel requires the use of pilots. The rapid variations of the channel require a minimum of channel knowledge by pilots in order to get a correct detection datum.

In this paper, we will focus on two aspects which influence strongly the channel estimation: pilot's geometry used and the algorithms applied to estimate the transfer function gains associated to data subcarriers. In the following section, we rely on the work of $[7,8]$, using a model of BEM (Basis Expansion Modeling) channel development. Then, we propose a pilot subcarriers configuration to improve the estimation for rapid changes in time channel. Our objective is to enhance the spectral efficiency with the same number of pilots in order to increase the data rate.

The paper is organized as follows. Section II formulates the data model for OFDM system. Section III pre- 
sents the chosen propagation channel model and its Basis Expansion Modeling (BEM) approach. In Section IV, this approach is applied to channel estimation with a dynamic allocation of pilot subcarriers. Simulation results are shown in Section V. Finally, we conclude the presented study in section VI.

Notation: $X$ is a real scalar, $X$ is a vector, $X$ is a matrice. $\boldsymbol{X}^{H}, \boldsymbol{X}^{+}, \boldsymbol{X}^{*}, \boldsymbol{X}^{T}$ represent respectively hermetian, opposite, conjugate and transpose of the matrice $\boldsymbol{X} . X(k)$ is the $k^{\text {th }}$ element of vector $X$ and $\boldsymbol{X}(i, k)$ are the $i^{\text {th }}$ and $k^{\text {th }}$ entries of matrice $\boldsymbol{X}$. $\otimes$ is the circular convolution operator.

\section{System Model}

The multipath effect introduced by the propagation channel causes interferences between symbols when using a transmission type series. A good solution to overcome the effects of the channel is to use a parallel or multicarrier transmission. The OFDM technique consists to convert the serial coded data stream on parallel blocks and to transmit simultaneously these blocks over several orthogonal carriers using the Inverse Fast Fourier Transform (IFFT). In the transmitter side, the data stream is grouped into blocks of $N$ data symbols, called OFDM symbol, and are represented by the vectors $X_{i}(k)(k, i$ : are frequency and OFDM symbol index). Then, an IFFT is performed on each data symbols block:

$$
S_{i}(t)=\frac{1}{N} \sum_{k=1}^{N} X_{i}(k) \cdot \mathrm{e}^{j 2 \pi \cdot f_{k} \cdot t},
$$

where $X_{i}(k)$ represents the complex data (M-PSK or M-QAM) which modulates the frequency $f_{k}$, and $f_{k}=k / T_{b} . T_{b}$ is the symbol OFDM duration. By sampling the continuous signal $S_{i}(t)$ at time sampling $T_{e}$ $\left(t=n \cdot T_{e}\right.$ and $\left.T_{b}=N \cdot T_{e}\right)$, we obtain the following equation:

$$
S_{i}(n)=\frac{1}{N} \sum_{k=1}^{N} X_{i}(k) \cdot \mathrm{e}^{j 2 \pi \cdot \frac{n \cdot k}{N}} .
$$

The received signal $R_{i}(n)$ is generally the sum of a convolution of the channel impulse response $h(n)$ with the signal $S_{i}(n)$ and an additive white Gaussian noise $V_{i}(n)$. It is implicitly assumed that the transmitter and the receiver are perfectly synchronized:

$$
R_{i}(n)=\sum_{l=1}^{L} h_{l}(n) \otimes S_{i}(n-l)+V_{i}(n),
$$

where $\otimes$ is the symbol of the circular convolution operator and $V_{i}(n)$ are samples of additive white gaussian noise (AWGN). At the receiver side, the cyclic prefix is removed and the data symbols $\boldsymbol{Y}$ are obtained by performing the FFT operation:

$$
\boldsymbol{Y}=\boldsymbol{H} \cdot \boldsymbol{X}+\boldsymbol{V}=\boldsymbol{F} \boldsymbol{H}^{t} \boldsymbol{F}^{H} \cdot \boldsymbol{X}+\boldsymbol{V},
$$

where $\boldsymbol{Y}=\boldsymbol{Y}(i, k), \quad \boldsymbol{X}=\boldsymbol{X}(i, k), \quad \boldsymbol{V}=\boldsymbol{V}(i, k), \quad \boldsymbol{H}^{t}$ and $\boldsymbol{H}$ represent the channel matrices respectively in time and frequency domain for each OFDM symbol. The channel matrix $\boldsymbol{H}$ is not diagonal because of the channel temporal variations during an OFDM symbol (i.e. $\boldsymbol{H}(k, z), \quad k$ transmitted and $z$ received carriers). It is expressed using the following equation $[9,10]$ :

$$
\boldsymbol{H}(k, z)=\frac{1}{\sqrt{N}} \sum_{n=1}^{N} \boldsymbol{H}^{t}(k, n) \cdot \mathrm{e}^{-j 2 \pi \frac{(k-z) n}{N}},
$$

where $\boldsymbol{H}^{t}(k, n)$ represents the Fourier transform of the impulse response $h_{l}(n)$ :

$$
\boldsymbol{H}^{t}(k, n)=\frac{1}{\sqrt{N}} \sum_{l=1}^{L} h_{l}(n) \cdot \mathrm{e}^{-j 2 \pi \frac{k l}{N}},
$$

with $L$ is the total number of paths in the multipath fading channel.

\section{Propagation Channel Model}

Since many years, different approaches for mobile radio channels modeling and simulation have been developed in the literature. But, no specific channel model is proposed for the mobile communications taking into account very high velocities such those encountered for very high speed train. The model of Clarke [11], which is the mathematical model of reference, and the simplified version of Jakes [12] are the most widely used to model variations in the signal channels called Rayleigh fading.

Simulation of a fading channel with Doppler calls for the generation of a Gaussian random process in order to mimic the sampled version of the channel waveform. These simulations are widely used in the process of designing efficient and robust next generation wireless communication and broadcasting systems. One important aspect to be considered is the accuracy of such simulation methods. Statistically, the provided discrete channel response samples should meet certain requirements related to the spectrum and time-domain variations. Hence, some quality measures have been introduced such as the probability density function (PDF), the cumulative distribution function (CDF), the autocovariance function (ACF), the level crossing rate (LCR) and the average duration of fades (ADF). Generated samples should meet the requirements in terms of quality measures during the predefined simulation period. The task of designing a channel simulator should tackle these issues while keeping a reasonable computation complexity and memory requirements.

State-of-the-art Rayleigh fading simulators can be divided into three main categories: the sum of sinusoids (SOS) method, filtering white Gaussian variables method and the inverse fast Fourier transform (IFFT) method. In [13], Pop and Beaulieu showed that the Jakes simulator 
is not wide-sense stationary in some cases and proposed other modifications. However, although the number of sinusoids is considered close to infinity, the higher order statistics of the simulator do not follow the theoretical statistical model of Clarke. This was also shown in [14,15]. In [16], Xiao and Zheng have proposed a new model for a sum of sinusoids Rayleigh fading channel that generates a non-deterministic signal with statistical properties converging to those of Jakes model even with a small number of sinusoids $N_{s}$.

The following equations give the fading process of this simulator:

$$
\begin{gathered}
Z(t)=Z_{c}(t)+j Z_{s}(t) \\
Z_{c}(t)=\frac{2}{\sqrt{N_{s}}} \sum_{n=1}^{N_{s}} \cos \left(\omega_{d} \cdot t \cdot \cos \alpha_{n}+\psi_{n}\right) \\
Z_{s}(t)=\frac{2}{\sqrt{N_{s}}} \sum_{n=1}^{N_{s}} \cos \left(\omega_{d} \cdot t \cdot \sin \alpha_{n}+\psi_{n}\right) \\
\alpha_{n}=\frac{2 \pi n-\pi+\theta}{4 N_{s}},
\end{gathered}
$$

where $\omega_{d}=2 \pi F_{d} ; \alpha_{n}, \psi_{n}$ and $\theta$ are random variables statistically independent and uniformly distributed (iid) on $[-\pi, \pi]$ for any number of sinusoids $N_{s} \cdot \alpha_{n}$, $\psi_{n}$ represent respectively the arrival angle in the direction of movement of the receiver and the initial phase.

This model is selected for the proposed system simulations presented in this paper. In the next section, we describe the approximation of the complex channel gains by a development in basis functions. The goal is to model the temporal channel variations during an OFDM symbol and to determine their evolutions during to the transmission duration.

\section{Basis Expansion Modeling}

The idea of Basis Expansion Modeling (BEM) is described in [8,17,18-22]. The use of such a development is particularly useful to reduce the number of channel parameters to be estimated on a symbol time. For a block transmission during $N$ symbols, the channel is characterized by $(N \times L)$ samples, $L$ is the total number of broadband channel paths according to the representation given by the relation (5) in which each path has a different realization at each time. The BEM model represents the total samples of the channel complex gains $(L \times N)$ with a minimum of basis function coefficients BEM $(l \times Q)$. In this model, each path of the channel $h_{l}(n)$ is represented by:

$$
h_{l}(n)=\boldsymbol{B} \cdot C b_{l}(n)+\varepsilon_{l}(n),
$$

where $\boldsymbol{B}$ is the matrix approximation of the basis functions and $Q$ is the order the BEM development.
$C b_{l}(n)=C b_{l}=\left[C b_{l, 0}, C b_{l, 1}, \cdots, C b_{l, Q-1}\right]^{\mathrm{T}}$ contains the $Q$ coefficients corresponding to the basic $N$ samples $h_{l}(n)$ and $\varepsilon_{l}$ is the modeling error that's often neglected and dependent on the number $Q$. For the basis function matrix $\boldsymbol{B}$, there are several approaches: the Discrete Spheroidal Sequences (DSS-BEM), the Karhunen-Loeve (KL-BEM), the Complex Exponential (CEBEM) or the Polynomial (P-BEM) [8,18,23-25]. Subsequently, we will focus on the P-BEM.

Considering the modeling error $\varepsilon_{l}$ equal to zero (i.e. $\left.h_{l}(n)=\boldsymbol{B} \cdot C b_{l}(n)\right)$, the correlation matrix of the basis coefficients $C b_{l}(n)=\boldsymbol{B}^{+} \cdot h_{l}(n)$ is defined by:

$$
\boldsymbol{R}_{C b_{l}}=\boldsymbol{B}^{+} \boldsymbol{R}_{h_{l}} \boldsymbol{B}^{+H} \text {. }
$$

Regarding the channel estimation that is modeled by a BEM development, we only need to estimate the basis coefficients. We chose to model the channel path by a PBEM [26]. In this case, $\boldsymbol{B}$ has the following expression $\boldsymbol{B}_{n, q}=n^{q}$ where $n=0, \cdots, N_{-1}$ and $q=0,1, \cdots, Q-1$.

$$
\boldsymbol{B}_{n, q}=\left[\begin{array}{cccc}
1 & 1 & \cdots & 1 \\
0^{1} & 1^{1} & \cdots & (N-1)^{1} \\
\vdots & \vdots & \ddots & \vdots \\
0^{Q-1} & 1^{Q-1} & \cdots & (N-1)^{Q-1}
\end{array}\right]^{\mathrm{T}}
$$

\section{Proposed Channel Estimation Technique Channel Estimation with Dynamic Allocation of Pilots}

In this section we consider the estimation of a timevarying channel with pilot subcarriers whose positions change from one OFDM symbol to another. We perform time channel estimation instead of the frequency estimation. Indeed, the last estimation based on conventional methods such as ML (Maximum Likelihood, MMSE (Minimum Mean Square Error) or LS (Least Square), gives poor performances because the estimated channel matrix is no longer diagonal in this context $[8,9,26]$.

We propose to vary the positions of the pilot carriers in the OFDM symbol, in order to scan the whole spectrum of the OFDM symbol (see Figure 1). The aim is, firstly, to avoid the estimation of the channel on the same pilots positions, which could be lost by fading, and secondly, to obtain an adaptive estimation of the channel. This is the proposed technique called Dynamic Allocation of Pilots (DAP).

Figure 2 illustrates the operating mechanism of the DAP. We note here that before applying the IFFT signal $X_{i}(k)$, it consists of complex payload $C_{i}(k)$ (QPSK or 16QAM) and pilots $P_{i}(k)$. The whole is bounded by guard bands $0_{i}(k)$ (Zero Padding).

In this context, we consider an expansion in the $\mathrm{P}$ - 


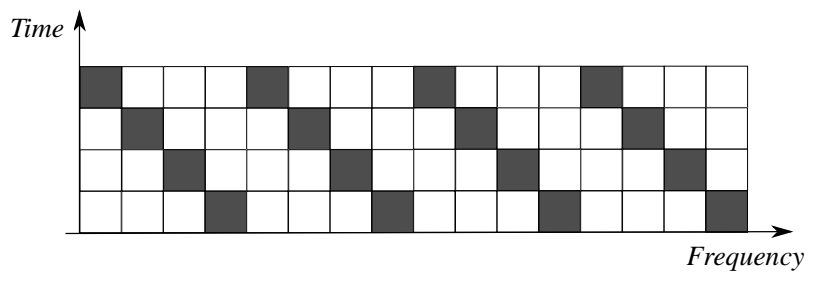

Data

Pilots

Figure 1. OFDM frame structure with pilot carrier offset.

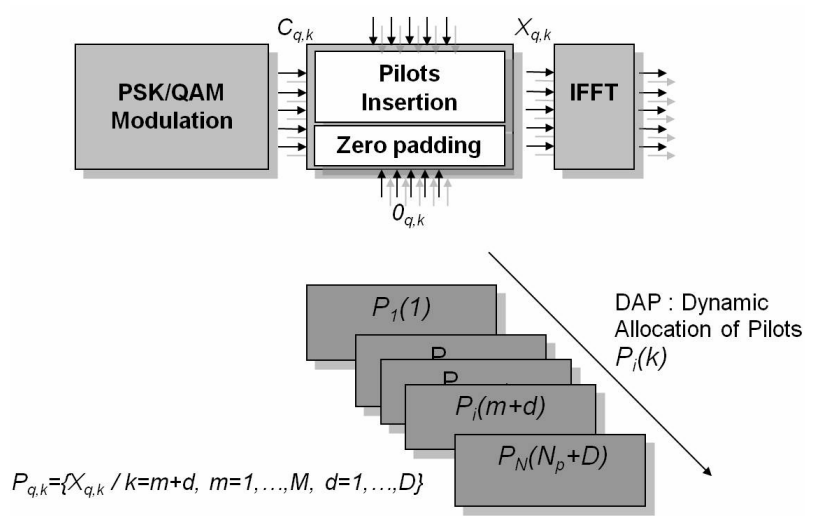

Figure 2. Block diagram of the DAP.

BEM basic functions for the channel model in order to reduce system complexity while maintaining an acceptable level of performance. Equation (14) can be rewritten as $\boldsymbol{Y}=\boldsymbol{W} \cdot \boldsymbol{h}+\boldsymbol{V} \quad[27,28]$. So the $i^{\text {th }}$ symbol can be written as:

$$
\begin{aligned}
Y_{i}(n) & =\frac{1}{N} \sum_{n=0}^{N-1} h(n) \cdot X_{i}(k) \cdot \mathrm{e}^{-j \frac{2 \pi n(i-k)}{N}}+V_{i}(n) \\
& =\frac{1}{N} \sum_{n=0}^{N-1} W_{i}(n) \cdot h(n)+V_{i}(n),
\end{aligned}
$$

or $W_{i}(n)=\sum_{k=0}^{N-1} \Omega_{i}(k, n) \cdot X_{i}(k)$ and

$\Omega_{i}(k, n)=\mathrm{e}^{-j \frac{2 \pi n(i-k)}{N}}$.

Assuming that $N_{p}$ is the number of pilot subcarriers placed at positions $\Psi=\left\{X(1), \cdots, X(m), \cdots, X\left(N_{p}\right)\right\}$, and we know $X(k=m)$, where $m \in\{k / k=1: D: N\}$, $m \in\left\{1, \cdots, N_{p}\right\}$ and $D$ is the space between two successive pilot subcarriers. It is then possible to reformulate $\boldsymbol{W}(i, n)$ as the sum of two matrices such as:

$$
\begin{aligned}
\boldsymbol{W}_{i}(n) & =\sum_{k=m}^{N-1} \Omega_{i}(k, n) \cdot X_{i}(k)+\sum_{k \neq m}^{N-1} \Omega_{i}(k, n) \cdot X_{i}(k) \\
& =\boldsymbol{W}_{i}^{k \in \Psi}(n)+\boldsymbol{W}_{i}^{k \notin \Psi}(n) \\
& =\boldsymbol{W}_{i}^{P}(n)+\boldsymbol{W}_{i}^{\text {data }}(n),
\end{aligned}
$$

where $\boldsymbol{W}_{i}^{P}(n)$ corresponds to the pilots and $\boldsymbol{W}_{i}^{\text {data }}(n)$ is the useful data.
By replacing $\boldsymbol{W}_{i}(n)$ in Equation (14), we obtain:

$$
\begin{aligned}
& Y_{i}(n)=\boldsymbol{W}_{i}^{P}(n) \cdot h+\boldsymbol{W}_{i}^{\text {data }}(n) \cdot h+V_{i}(n) \\
& \text { or } Y_{i}=\boldsymbol{W}_{i}^{P} \cdot h+\boldsymbol{W}_{i}^{\text {data }} \cdot h+V_{i} .
\end{aligned}
$$

Now, we will use the pilot positions to estimate the channel impulse response $h$. Let

$\tilde{Y}=\left[Y_{i}(1), \cdots, Y_{i}(m), \cdots, Y_{i}\left(N_{p}\right)\right]^{\mathrm{T}}$ and

$\tilde{V}=\left[V_{i}(1), \cdots, V_{i}(m), \cdots, V_{i}\left(N_{p}\right)\right]^{\mathrm{T}}$ corresponding to the positions of the pilots. We can build $N_{p}$ linear equations using the following equation:

$$
\begin{aligned}
\left(\begin{array}{c}
Y_{i}(1) \\
\vdots \\
Y_{i}\left(N_{p}\right)
\end{array}\right) & \left(\begin{array}{ccc}
W_{i}^{P}(1,0) & \ldots & W_{i}^{P}(1, N \cdot L-1) \\
\vdots & \ddots & \vdots \\
W_{i}^{P}\left(N_{p}, 0\right) & \cdots & W_{i}^{P}\left(N_{p}, N \cdot L-1\right)
\end{array}\right) \cdot h \\
& +\left(\begin{array}{c}
V_{i}(1) \\
\vdots \\
V_{i}\left(N_{p}\right)
\end{array}\right)
\end{aligned}
$$

or

$$
\tilde{Y}=\tilde{W} \cdot h+\tilde{V} .
$$

To find the impulse response $h$ as a least squares solution of the system, we'll replace it with its basis coefficients $C b$. Omitting the indices, the Equation (18) becomes $[27,28]$ :

$$
\tilde{Y}=\tilde{W} \cdot B \cdot C b+\tilde{V},
$$

hence the expression of the estimated channel $h$ becomes:

$$
\hat{h}=\boldsymbol{B} \cdot C b=\boldsymbol{B}(\tilde{\boldsymbol{W}}, \boldsymbol{B})^{+} \tilde{Y} .
$$

\section{Simulation Results}

In the following, we consider an OFDM system. The total number of subcarriers is 128 in which subcarriers are deployed for data transmission and subcarriers are used like pilots. We set to zero the middle DC carrier and the lateral subcarriers placed on left and on right of the OFDM symbol spectrum. A Cyclic Prefix (CP) with samples is used and the OFDM symbol duration is $72 \mu \mathrm{s}$. For the channel model simulation, we have chosen the Zheng model with a Jakes spectrum for mobile radio channel with respect to the motion between the transmitter and the receiver. Assuming that the synchronization between the transmitter and the receiver is perfect at receiver, the Bit Error Rate (BER) is evaluated for three normalized Doppler frequencies $F_{1}=0.0003, F_{2}=$ 0.0006 and $F_{3}=0.0008$ corresponding to a mobile speed $v$ of 140,280 and $350 \mathrm{kmph}$ at a carrier frequency $f_{0}=2.5$ $\mathrm{GHz}$. 
Figures 3 and 4 illustrate the evolution of BER with increasing the value of the normalized Doppler frequency. Concerning the QPSK modulation, in a channel where the temporal variations are quantified by $F_{1}$ and $F_{2}$ and energy per bit $\frac{E_{b}}{N_{o}} \geq 25 d B$, The DAP improvement is about 1 or $2 \mathrm{~dB}$. The DAP proposed method tracks channel variations better than the conventional case with fixed pilots. On the other hand, for the value $F_{3}(350$ $\mathrm{kmpl}$ ), the DAP offers a lower gain of $0.5 \mathrm{~dB}$. This is explained by the rapid variations in the channel and changing the pilot positions doesn't achieve the same performance as the values for $F_{1}$ and $F_{2}$.

Concerning the 16 QAM and for three values of the normalized Doppler frequency, the effect of mobility on performance is significant. The DAP is limited by a gain

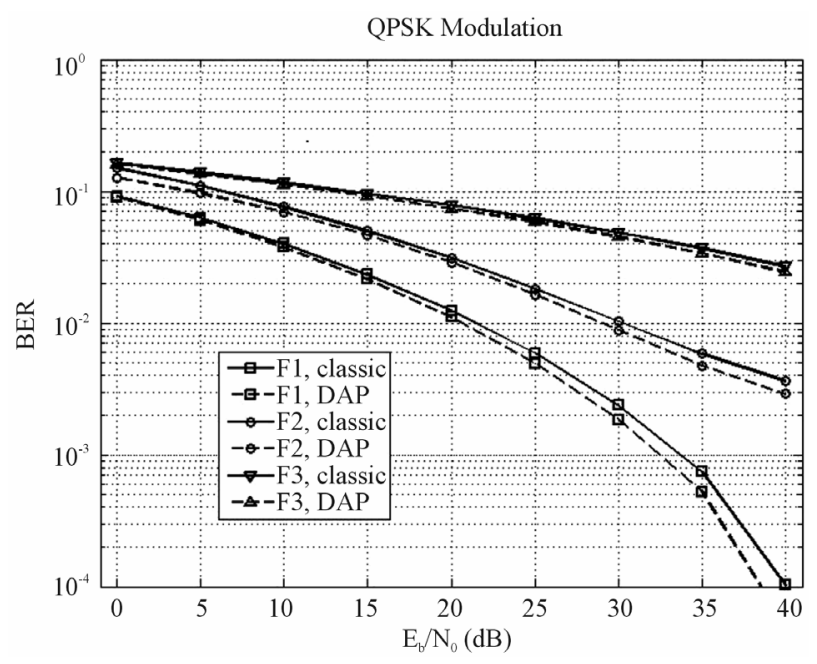

Figure 3. Comparison of classical and DAP channel estimations for QPSK modulation.

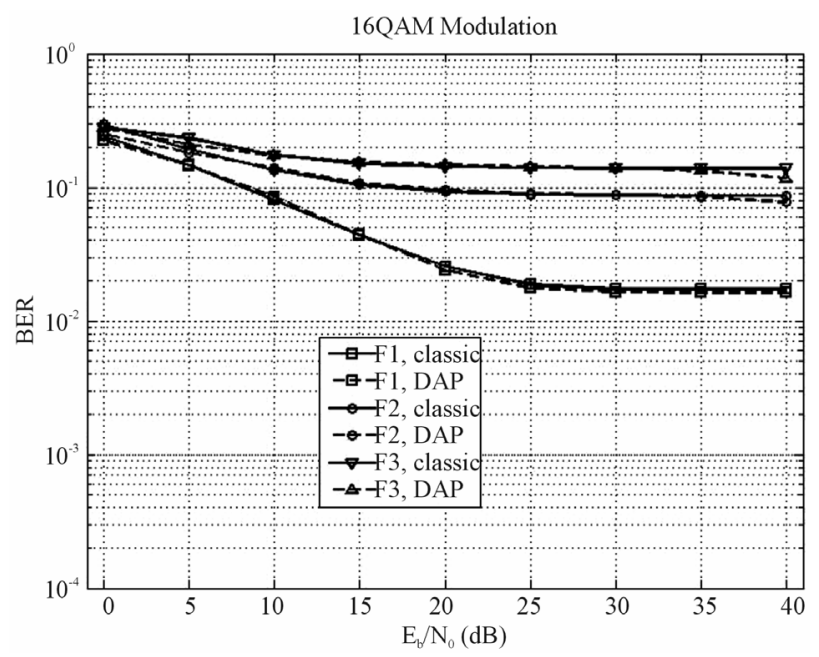

Figure 4. Comparison of classical and DAP channel estimations for 16 QAM modulation. which does not exceed $0.25 \mathrm{~dB}$. This can be due to the size of the constellation of 16 QAM.

\section{Conclusion}

In this paper we proposed a solution to minimize the Doppler Effect damage and consequence on the performances of OFDM communication systems. OFDM technology provides efficient estimation of time-variant channels. In order to estimate the channel, pilot subcarriers are inserted into the OFDM symbol. The channel is estimated in the time domain and the temporal variations of each path are modeled by BEM. A pilot structure based on varying progressively the positions of pilot subcarriers from a symbol to another, is proposed. The pilot subcarrier positions are allocated dynamically during the transmission of the OFDM symbols with the DAP. The estimated time-varying channel is better and therefore the system performance will be improved in presence of the Doppler Effect. Using the DAP, the results show that the time-varying channel estimation is improved and the severe consequences of the Doppler Effect are reduced.

\section{Acknowledgements}

We thank the International Campus on Safety and Intermodal Transportation program (CISIT), French National Research Agency ANR and Transports Terrestrial Promotion Northern France (I-Trans).

\section{REFERENCES}

[1] L. Hanzo, M. Munster, B. Choi and T. Keller, "OFDM and MCCDMA for Broadband Multi-User Communications, WLANs and Broadcasting," John Wiley and Sons, Hoboken, 2003.

http://dx.doi.org/10.1002/9780470861813

[2] I. Bradaric and A. P. Petropulu, "Performance of Training-Based OFDM Systems in the Presence of Time Varying Frequency Selective Channels," IEEE International Conference on Acoustics, Speech, and Signal Processing, Vol. 4, No. 29, 2004, pp. 737-740.

[3] X. Cai and A. N. Akansu, "A Subspace Method for Blind Channel Identification in OFDM Systems," IEEE International Conference on Communications, New Orleans, 18-22 June 2000, pp. 929-933.

[4] M. de Courville, "Utilisation de Bases Orthogonales pour l'Algorithmique Adaptative et l'Égalisation des Systèmes Multiporteuses," Ph.D. Thesis, École Nationale Supérieure de Télé-communications, Rennes, 1996.

[5] R. W. Heath and G. B. Giannakis, "Blind Channel IdentiFication for Multirate Precoding and OFDM Systems," Proceedings of 13th International Conference on Digital Signal Processing, Santorini, 2-4 July 1997, pp. 383-386. http://dx.doi.org/10.1109/ICDSP.1997.628115

[6] T. Yamamura and H. Harada, "High Mobility OFDM 
Transmission System by a New Channel Estimation and ISI Cancellation Scheme Using Characteristics of Pilot Symbol Inserted OFDM Signal," Proceedings of IEEE 50th Vehicular Technology Conference, Amsterdam, 1922 September 1999, pp. 319-323.

[7] Z. Tang, G. Leus, R. C. Cannizzaro and P. Banelli, "Pilot Assisted Time-Varying OFDM Channel Estimation," IEEE Transactions on Signal Processing, Vol. 55, No. 5, 2006, pp. 2226-2238.

http://dx.doi.org/10.1109/TSP.2007.893198

[8] T. Tian, D. Gang and J. Jun, "Estimation of Time Varying Channels for Pilot Assisted OFDM Systems," Journal of China Universities of Posts and Telecommunications, Vol. 14, No. 2, 2007, pp. 94-99. http://dx.doi.org/10.1016/S1005-8885(07)60133-2

[9] R. C. Cannizzaro, P. Banelli and G. Leus, "Adaptive Channel Estimation for OFDM Systems with Doppler spread," Proceedings of IEEE Conference on Signal Processing Advances in Wireless Communications, Cannes, 2-5 July 2006, pp. 1-5.

[10] S. Sezginer and Y. El Hajj Shehadeh, "An Iterative Channel Estimator for Fast-Varying Channels Using Successive OFDM Symbols," IEEE 20th International Symposium on Personal, Indoor and Mobile Radio Communications, Tokyo, 13-16 September 2009, pp. 2404-2408.

[11] R. H. Clarke, "A Statistical Theory of Mobile Radio Reception," Bell System Technical Journal, Vol. 47, No. 6, 1968, pp. 957-1000. http://dx.doi.org/10.1002/j.1538-7305.1968.tb00069.x

[12] W. C. Jakes, "Microwave Mobile Communications," 2nd Edition, Wiley-IEEE Press, 1994. http://dx.doi.org/10.1109/9780470545287

[13] M. F. Pop and N. C. Beaulieu, "Limitations of Sum-of Sinusoids Fading Channel Simulators," IEEE Transactions on Communications, Vol. 49, No. 4, 2001, pp. 699708. http://dx.doi.org/10.1109/26.917776

[14] P. Dent, G. E. Bottomley and T. Croft, "Jakes Fading Model Revisited," IEEE Electronics Letters, Vol. 29, No. 13, 1993, pp. 1162-1163. http://dx.doi.org/10.1049/el:19930777

[15] S. A. Fechtel, "A Novel Approach to Modeling and Efficient Simulation of Frequency-Selective Fading Radio Channels," IEEE Journal on Selected Areas in Communications, Vol. 11, No. 3, 1993, pp. 422-431. http://dx.doi.org/10.1109/49.219555

[16] C. Xiao, Y. R. Zheng and N. C. Beaulieu, "Novel Sum-of Sinusoids Simulation Models for Rayleigh and Rician Fading Channels," IEEE Transactions on Wireless Communications, Vol. 5, No. 12, 2006, pp. 3667-3679. http://dx.doi.org/10.1109/TWC.2006.256990

[17] P. Banelli, R. Cannizzaro and L. Rugini, "Data Aided Kalman Tracking for Channel Estimation in Doppler Affected OFDM Systems," Proceedings of IEEE International Conference on Acoustics, Speech and Signal Processing, Honolulu, 15-20 April 2007, pp. 133-136.
[18] H. Senol, H. Cirpan and E. Panayirci, "A Low Complexity KL Expansion Based Channel Estimator for OFDM Systems," EURASIP Journal on Wireless Communications and Networking, Vol. 2005, No. 2, 2005, pp. 136-174.

[19] Z. Tang, G. Leus and P. Banelli, "Pilot-Assisted TimeVarying OFDM Channel Estimation Based on Multiple OFDM Symbols," Proceedings of IEEE 7th Workshop on Signal Processing Advances in Wireless Communications, Cannes, 2-5 July 2006, pp. 1-5.

[20] S. Tomasin, A. Gorokhov, H. Yang and J. P. Linnartz, "Iterative Interference Cancellation and Channel Estimation for Mobile OFDM," IEEE Transactions on Wireless Communications, Vol. 4, No. 1, 2005, pp. 238-245. http://dx.doi.org/10.1109/TWC.2004.840194

[21] B. Yang, K. Letaief, R. Cheng and Z. Cao, "Channel Estimation for OFDM Transmission in Multipath Fading Channels Based on Parametric Channel Modeling," IEEE Transactions on Communications, Vol. 49, No. 3, 2001, pp. 467-479. http://dx.doi.org/10.1109/26.911454

[22] G. B. Giannakis and C. Tepedelenlioglu, "Basis Expansion Models and Diversity Techniques for Blind Identification and Equalization of Time-Varying Channels," Proceedings of the IEEE, Vol. 86, No. 10. 1998, pp. 19691986. http://dx.doi.org/10.1109/5.720248

[23] T. Whitworth, M. Ghogho and D. McLernon, "Optimized Training and Basis Expansion Model Parameters for Doubly-Selective Channel Estimation," IEEE Transactions on Wireless Communications, Vol. 8, No. 3, 2009, pp. 1490-1498.

http://dx.doi.org/10.1109/TWC.2009.080364

[24] T. Zemen and C. F. Mecklenbrauker, "Time-Variant Channel Estimation Using Discrete Prolate Spheroidal Sequences," IEEE Transactions on Signal Processing, Vol. 53, No. 9, 2005, pp. 3597-3607. http://dx.doi.org/10.1109/TSP.2005.853104

[25] H. Hijazi and L. Ros, "OFDM High Speed Channel Complexgains Estimation Using Kalman Filter and QR-Detector," IIEEE International Symposium on Wireless Communication Systems, Reykjavik, 21-24 October 2008, pp. 26-30.

[26] H. Hijazi and L. Ros, "Joint Data QR-Detection and Kalman Estimation for OFDM Time-Varying Rayleigh Channel Complex Gains," IEEE Transactions on Communications, Vol. 58, No. 1, 2010, pp. 170-178. http://dx.doi.org/10.1109/TCOMM.2010.01.080296

[27] W.-G. Song and J.-T. Lim, "Pilot-Symbol Aided Channel Estimation for OFDM with Fast Fading Channels," IEEE Transactions on Broadcasting, Vol. 49, No. 4, 2003, pp. 398-402. http://dx.doi.org/10.1109/TBC.2003.819049

[28] W.-G. Song and J.-T. Lim, "Channel Estimation and Signal Detection for MIMO-OFDM with Time Varying Channels," IEEE Communications Letters, Vol. 10, No. 7, 2006, pp. 540-542. 